Introduction The rising incidence of IBD, young age of onset and chronic nature mean that IBD has significant cost implications with the National IBD Audit estimating that cost to the National Health Service (NHS) exceeded $£ 1$ billion in 2010. The recent introduction of Clinical Commissioning Groups has also changed the way in which healthcare is paid for. This model is designed to be used by both commissioners and individual gastroenterology units to calculate the annual cost per patient of treating Ulcerative Colitis (UC) and Crohns Disease (CD) and to enable areas of potential cost savings to be explored.

Methods The cost of care for IBD was calculated by summing the costs of treatment, treatment side effects and disease-related complications, accounting for the proportions of patients incurring these costs. Default input values for costs, the percentage of patients receiving each treatment, and the percentage of patients experiencing side effects or complications were determined from sources such as the British National Formulary (BNF), National Institute for Clinical Excellence (NICE), NHS trusts and published literature. However, an important feature of the model was its customisability allowing users to input local data, thereby generating costs which were unique and precise for that unit.

Results Using default input values, the annual cost of treating any UC patient was estimated to be $£ 3,084$. For a UC patient in remission, in relapse with mild-to-moderate UC or in relapse with severe UC, annual cost per patient was estimated to be $£ 1,693, £ 2,903$ and $£ 10,760$, respectively. The annual cost for any $C D$ patient was estimated to be $£ 6,156$ ( $£ 1,800$ for patients in remission; $£ 10,513$ for patients in relapse). However, inputting local data would show some variability in the costs from trust to trust.

Annually $£ 743.65$ was spent per UC patient on mesalazines. The model allows exploration of the cost savings if the percentage of patients on each brand of mesalazine was altered.

When the percentage of relapsing CD patients on adalimumab was increased from $5 \%$ to $10 \%$, the annual cost per relapsing $C D$ patient rose from $£ 10,513$ to $£ 11,032$. The overall annual cost for any CD patient rose from $£ 6,156$ to $£ 6,416$.

Increasing the percentage of mild-to-moderate UC patients on leukapheresis from 0.5 to $8 \%$ increased the annual cost per mild-to-moderate patient from $£ 2,903$ to $£ 3,352$, and the annual cost for any UC patient from $£ 3,083$ to $£ 3,263$. However, assuming that increased use of leukapheresis would cause a decrease from $20 \%$ to $15 \%$ in the annual proportion of patients experiencing acute severe flares, the annual cost for any UC patient fell to $£ 3,078$.

Conclusion This model facilitates calculation of local annual costs per UC and CD patient, and allows areas to be identified where savings could be made.

Disclosure of Interest: None Declared.

\section{PTH-060 EVALUATION OF AN ESTABLISHED COMMUNITY GASTROENTEROLOGY CLINIC WITH COMPARISON TO SECONDARY CARE}

${ }^{1} \mathrm{OF}$ Ahmad*, ${ }^{2} \mathrm{R}$ Dawood, ${ }^{3} \mathrm{C}$ Oesterling, ${ }^{2} \mathrm{D}$ Rusk, ${ }^{4} \mathrm{~A}$ Akbar. ${ }^{1}$ Gastroenterology, Whittington Health, St Mark's Hospital, London, UK; ${ }^{2}$ NHS Harrow, St Mark's Hospital, London, UK; ${ }^{3}$ NHS Ealing, St Mark's Hospital, London, UK; ${ }^{4}$ Gastroenterology, St Mark's Hospital, London, UK

\subsection{6/gutjnl-2014-307263.506}

Introduction Community based specialist clinics improve patient experience and access to healthcare whilst improving GP-Specialist communication. ${ }^{1}$ Commissioning dictates that future management of chronic gastro-intestinal conditions needs to involve integrated care. We reviewed the outcomes of an established gastroenterology community clinic run for 3 years by two General Practitioners with a specialist interest in gastroenterology (GPwSIs) and a consultant gastroenterologist from the local hospital.

Methods A weekly gastroenterology community clinic in primary care was established to service 35 local General Practices. Data was collected from January-September 2013 using referral letters and an electronic database and compared to the local hospital general gastroenterology clinics.

Results Of 490 patients referred 284 (58\%) were triaged to community, 188 (38\%) referred onwards to secondary care and $18(4 \%)$ were returned to the referring GP. 37\% of appointments were conducted by the consultant.

The proportion of patients that did not attend was lower in the community (10\%) than secondary care (22\%). 86\% were discharged back to their referring GP; $16 \%$ after the $1^{\text {st }}$ appointment and $84 \%$ after one further follow up appointment. Others were directly referred to relevant secondary care. $80 \%$ had further investigations (39\% Gastroscopy, 24\% Colonoscopy, 22\% CT scan, 12\% Ultrasound abdomen, 9\% flexible sigmoidoscopy, $1 \%$ Oesophageal manometry) all performed in the local hospital.

Median wait time from referral to $1^{\text {st }}$ clinic appointment was 28 days in the community (56 days in the local hospital). Intraclinic wait time in community was an average of $3 \mathrm{~min}(38 \mathrm{~min}$ in secondary care). A community patient satisfaction survey $(\mathrm{N}=102)$ revealed that $88 \%$ would definitely recommend the clinic to a friend, with high levels of satisfaction. A secondary care satisfaction survey $(\mathrm{N}=214)$ showed that although overall satisfaction was similar, there was relative dissatisfaction of waiting times, waiting area and communication.

Conclusion This novel gastroenterology community clinic delivers high quality care closer to patients. It is associated with shorter refe, UKrral waiting times, high discharge rates and excellent patient satisfaction scores. Additional benefits include enhancement of primary/secondary care links, mentoring and teaching of GPwSIs, continuity of care and direct access to onward referral to secondary care for further management across specialties. Further follow up is necessary to evaluate the effect on local healthcare delivery.

\section{REFERENCE}

1 Powell J. Systematic review of outreach clinics in primary care in the UK. J Health Serv Res Policy 2002:7:177-83

Disclosure of Interest None Declared.

\section{PTH-061 THE MANAGEMENT OF ACUTE UPPER GASTROINTESTINAL BLEEDING IN PAEDIATRIC PRACTICE: A NATIONAL SURVEY}

$1,{ }^{2} \mathrm{P}$ Henderson, ${ }^{2} \mathrm{R}$ Hansen, ${ }^{2} \mathrm{RK}$ Russell, ${ }^{2} \mathrm{P}$ McGrogan. ${ }^{1}$ Child Life and Health, University of Edinburgh, Edinburgh, UK; ${ }^{2}$ Paediatric Gastroenterology and Nutrition, Royal Hospital for Sick Children, Glasgow, UK

10.1136/gutjnl-2014-307263.507

Introduction Acute upper gastrointestinal (GI) haemorrhage is rare in paediatric practice but continues to cause significant morbidity and mortality. Guidelines for the management of GI bleeding in children exist but their evidence base is limited and their implementation in the UK is unknown. We aimed to determine the provision for paediatric upper GI bleeding in the UK with regard to access to services and management using a national survey. 
Methods In August 2013 an electronic survey containing 20 questions was sent to the $18 \mathrm{RCPCH}$-approved National GRID paediatric gastroenterology training centres who also act as regional referral centres. The questionnaire was completed online by either the departmental clinical or endoscopy lead and results collated by the study centre. Descriptive statistics were used to present results.

Results Sixteen centres responded, representing the experience of 65.6 whole-time equivalent (WTE) consultant paediatric gastroenterologists. Half of centres provided out-of-hours cover for GI emergencies in their region, with surgeons providing the majority (69\%) of out-of-hours support, often in conjunction with GI specialists (44\%), for acute upper GI bleeds. 11/16 centres dealt with $<20$ endoscopies for upper GI bleeding annually with 3 centres dealing with $>40 /$ year. $63 \%$ of tertiary centres had a GI haemorrhage protocol available in electronic format (online/shared-drive), but $53 \%$ were not aware of a similar protocol in their respective DGHs; only $31 \%$ of centres provided 'at-risk' patients with emergency cards/advice. $88 \%$ of centres had access to interventional radiology in-hours and 63\% out-of-hours. The injection of vasoconstrictors/sclerosants/thrombotic agents and endoclips were available in all but one centre, however only $19 \%$ of centres (with between 3-4.6 WTE consultants) stated that all their consultants were competent in managing upper GI bleeds, with the same percentage stating that no consultant was competent in management; very few procedures were carried out by trainees. Only half of centres were undertaking regular case review of paediatric bleeding cases. All respondents were keen to be involved in a detailed review of UK practice.

Conclusion Our national survey of tertiary paediatric GI unit experiences' of acute upper GI bleeding demonstrates that a large proportion of centres do not have protocols in place in their own centre or referring units, with most centres performing fewer than 20 therapeutic endoscopic procedures for upper GI bleeding annually. The majority of centres have a small number of consultants competent in upper GI haemorrhage management with limited opportunities for training. There is also limited development of managed clinical networks that would allow more ready access to expert endoscopy provision.

Disclosure of Interest None Declared.

\section{PTH-062 EMERGENCY ADMISSIONS FOR ALCOHOL RELATED CONDITIONS: MAKING SENSE OF ROUTINE DATA}

${ }^{1} \mathrm{P}$ Lekharaju*, ${ }^{2} \mathrm{E}$ Thompson, ${ }^{3} \mathrm{M}$ Shawihdi, ${ }^{2} \mathrm{M}$ Pearson, ${ }^{1} \mathrm{~S}$ Hood, ${ }^{3} \mathrm{~K}$ Bodger. ${ }^{1}$ Dept of Gastroenterology, Aintree University Hospital, University of Liverpool, Liverpool, UK; ${ }^{2}$ Aintree Health Outcome Partnership, University of Liverpool, Liverpool, UK; ${ }^{3}$ Department of Gastroenterology, Institute of Translational Medicine, University of Liverpool, Liverpool, UK

\subsection{6/gutjnl-2014-307263.508}

Introduction Alcohol-related emergency admissions (ARA) are a major and rising hospital burden, resulting from conditions that range from short-term toxicity to end-stage organ damage, notably liver disease. We report a project to develop analyses of routine coding data, with a particular focus on metrics related to 'frequent flyers' (FFs) as targets for new service interventions.

Methods Sources of data: Hospital Episode Statistics for all English hospitals (2006-2008); Inpatient coding data and AED attendances for our Trust (2006-2013).

Analysis: Screening of all non-emergency episodes for alcohol-related codes at any position to flag ARAs; linkage of individual cases to extract all admissions and order chronologically; allocation of each admission to a category based on primary and lower order diagnoses (flagging LIVER and NON-LIVER admissions); identification of frequent flyers (FFs) based on various definitions of admission count; linkage of ARAs to all-cause AED attendances in local data; funnel-plot analyses of patterns across English Trusts; longitudinal trends in local data.

Results Nationally: 219,158 ARAs in 139,077 patients (20062008), mean age (sd): 49.5 yrs (16); males: 99,271 (71\%); Deprivation: Quintile 1 (most deprived) 36.4\%, Quintile 5 (least): 9.4\%; Co-morbidity (Charlson), 0.44 (0.68); LoS: 7 (14) days; Inpatient death: 6,656 (4.8\%). No. admissions ranged from 160 per individual. Frequent Flyers: In two years, a cut-off of $5+$ admissions identified 5,404 FFs (4\% of patients; accounting for $18 \%$ of ARAs nationally) whereas $10+$ identified 909 FFs (1\% of patients; $6 \%$ of ARAs). Mean ARAs per Trust was 927 (range: $235-3930)$ with 6 -fold variation in $\%$ of FFs $(1.3 \%$ to $7.7 \%)$ and 4-fold variation in \% with liver disease across English hospitals (range: 7.6-30.2\%). As expected, FFs coded with liver disease had significantly higher LoS and mortality risk consistent with end-stage organ damage and "unavoidable" admissions.

Locally: 21,308 ARAs in 16,305 patients (2006-2012), with annual number of cases rising from 1,615 to 4,603. Defining FFs as $5+$ ARAs per year, there were 320 FFs (2\% of patients; $10 \%$ of ARAs). There was a year-on-year rise in ARAs $(2,454$ $5,510)$ and AED attendances without admission $(2,499-5,979)$. However, FFs ( $5+$ admissions) declined from 64 to 47 between 2006 and 2012 and non-liver FFs from 25 to 12, suggesting a positive impact of new local services on multiple attenders, especially those lacking established liver disease.

Conclusion We have developed a set of candidate metrics focussed on FFs, short stays and selected baskets of conditions to provide data to support front-line acute alcohol services.

Disclosure of Interest None Declared.

\section{PTH-063 IS EVENING ENDOSCOPY COST EFFECTIVE?}

P Closier*, C Hollywood. Gloucestershire Royal Hospital, Gloucester, UK

\subsection{6/gutjnl-2014-307263.509}

Introduction Currently in the UK we are seeing an increasing demand for endoscopy services that is set to continue to rise over the coming years. ${ }^{1}$ Our aim as an endoscopy department is to deliver a patient focused service, and patient choice is an integral component of this. The option of evening endoscopy lists is a novel approach to widening patient choice for provided services whilst simultaneously working towards a 7 day working week. Our aim was to evaluate the success of evening lists in terms of attendance and endoscopic outcomes to ensure the most cost effective evening service whilst identifying potential areas for development.

Methods We analysed data for scheduled evening endoscopy sessions (18.00 to 21.00) booked at Gloucestershire Royal Hospital over a 3 month period from April 2013. Information about preendoscopy fast, endoscopic results and non-attendance (DNA) rates was processed.

Results 54 patients were listed for endoscopy, of these 18 (33\%) endoscopies were inconclusive; 6 (35\%) abandoned due to food bolus, with 9 (53\%) non-attenders. Food bolus patients had fasting times between 6-8 $\mathrm{h}$ (as per protocol), but all had taken a substantial meal pre-fasting and 50\% were diabetic. All nonattenders were booked for variceal surveillance.

Conclusion Developing evening services is essential to meet user needs; however more guidance is needed to ensure cost effectiveness for the trust. In order to run the most efficient service 